\title{
Research and develop a management and control system of sports fitness promenade
}

\author{
Wang yong ${ }^{1, \mathrm{a}}$, Zhou hu $^{2, \mathrm{~b}}$, Pan yuexian ${ }^{3, \mathrm{c}}$, Xiong tao ${ }^{4, \mathrm{~d}}$, Zhang hui ${ }^{5, \mathrm{e}}$ \\ ${ }_{1,3,4,5}$ College of sportunterricht, Donghua University, 201620, China \\ ${ }^{2}$ College of Mechanical Engineering, Donghua University, 201620, China \\ awangyong@dhu.edu.cn, ${ }^{b}$ tigerzhou@dhu.edu.cn, ${ }^{\mathrm{c}} \mathrm{pyx} @$ dhu.edu.cn, ${ }^{\mathrm{d}} \mathrm{xiongtao@dhu.edu.cn,}$ \\ ezhanghui1230@dhu.edu.cn
}

KEY WORDS: Sports fitness promenade; data acquisition; physique monitoring; RS485 network

\begin{abstract}
This paper introduces the necessity of sunshine sports fitness promenade construction. In order to real-time acquire the students' physical training process data, sensors are installed at each monitoring sites and RS485 network was constructed. The students' physique monitoring and analysis system are developed which improved the students' enthusiasm to participate in physical exercise effectively and laid the foundation for guiding students to improve physical quality.
\end{abstract}

\section{Introduction}

Physical and mental health of the teenagers, strong willed, full of vitality, is a manifestation of national vitality, also is a symbol of progress of a civilization society, and an important aspect of national comprehensive strength. In recent years, the youth sports enterprise developed vigorously, made great achievements in the work of school sports, nutrition level and morphological level of young people have improved continuously, which greatly improved the health quality of the whole people. However, we must see soberly that the society and schools tend to pay more attention to intellectual education than sports education because of the influence from over pursuit of culture and scientific education which lead to the overweight schoolwork burden and serious insufficient exercise time to students. Additionally, due to lack of sports facilities and conditions, the sports curriculum and sports activities for students could not be ensured. Recent health monitoring reports show that adolescents' physical index, strength endurance as well as speed declined continuously. Meanwhile, the poor eyesight rate remains high and the number of overweight boys and girls increased significantly in the city.

Sunshine Sports Fitness promenade is a new physical training project which is comprehensive, public, scientific, practical, challenging, interesting and safe for students to exercise. However, the existing fitness promenade lacks the function of gathering students' exercise data automatically, so teachers are unable to give scientific assessment of the students' physical quality and exercise guidance. In this paper, integration of management and control system of fitness promenade was put forwarded and developed, make its'operation more scientific and more efficient.

\section{Sunshine sports fitness promenade function description}

"Fitness promenade" designs the training program scientifically and comprehensively, including strength, speed, coordination, bounce quality training, take the interest and entertainment into consideration during exercise, makes the trainees in the game enjoy the fun of sports exercise, enhance physique. The selected projects are comprehensive body exercise, including hands, eyes, feet requirements, a full range of cooperation to complete the exercise, so the trainees must pay more attention to the overall development of the body. Fitness promenade generally use the closed combined exercise equipment, each distinct item focuses on different characteristics of physical exercise, and assessment can be carried out. Table 1 lists the sub project function. 
Table 1. The main exercise program and function of the "closed students sports fitness promenade"

\begin{tabular}{|c|c|c|}
\hline $\begin{array}{c}\text { Serial } \\
\text { number }\end{array}$ & Exercise project & The main training function \\
\hline 1 & uphill and downhill run & $\begin{array}{l}\text { The development of lower limbs kicking ability, improve } \\
\text { the strength of lower limbs }\end{array}$ \\
\hline 2 & The high jump & $\begin{array}{l}\text { improve the strength of lower limbs , Improve ankle } \\
\text { strength, improve the ability of lower limbs deep jump }\end{array}$ \\
\hline 3 & $\begin{array}{l}\text { Parallel ladder (male) } \\
\text { Catch } \\
\text { the step forward (female) }\end{array}$ & $\begin{array}{l}\text { Enhanced upper limbs muscle endurance, promote } \\
\text { shoulder, hip flexibility, improve the body balance ability }\end{array}$ \\
\hline 4 & Snake run & $\begin{array}{l}\text { Flexibility, coordination development of the } \\
\text { body, improve the ability to balance of the body in the } \\
\text { change to the sprint }\end{array}$ \\
\hline 5 & Obstacle crossing jump & $\begin{array}{l}\text { Improve the ability of the body over the obstacle in } \\
\text { running, improve physical coordination, flexibility }\end{array}$ \\
\hline 6 & Soft tennis climbing & $\begin{array}{l}\text { Improve the upper limb strength, development of people } \\
\text { climbing ability, enhance the body's coordination and } \\
\text { flexibility }\end{array}$ \\
\hline 7 & The swing bridge & $\begin{array}{l}\text { Increase the body's ability to maintain equilibrium in an } \\
\text { unstable conditions, develop the body's } \\
\text { flexibility, coordination }\end{array}$ \\
\hline 8 & The plum blossom piles & $\begin{array}{l}\text { Develop the hole body's flexibility, coordination improve } \\
\text { body's balance ability }\end{array}$ \\
\hline 9 & Support jump & $\begin{array}{l}\text { To improve the strength and coordination of upper } \\
\text { limb, lower limb, waist }\end{array}$ \\
\hline 10 & Through the tunnel & $\begin{array}{l}\text { Improve the waist and abdominal and lower limb } \\
\text { strength ,exercise control in the lower center of gravity } \\
\text { of body }\end{array}$ \\
\hline
\end{tabular}

Fig. 1 is the Sketch Map of Closed sports fitness promenade layout.

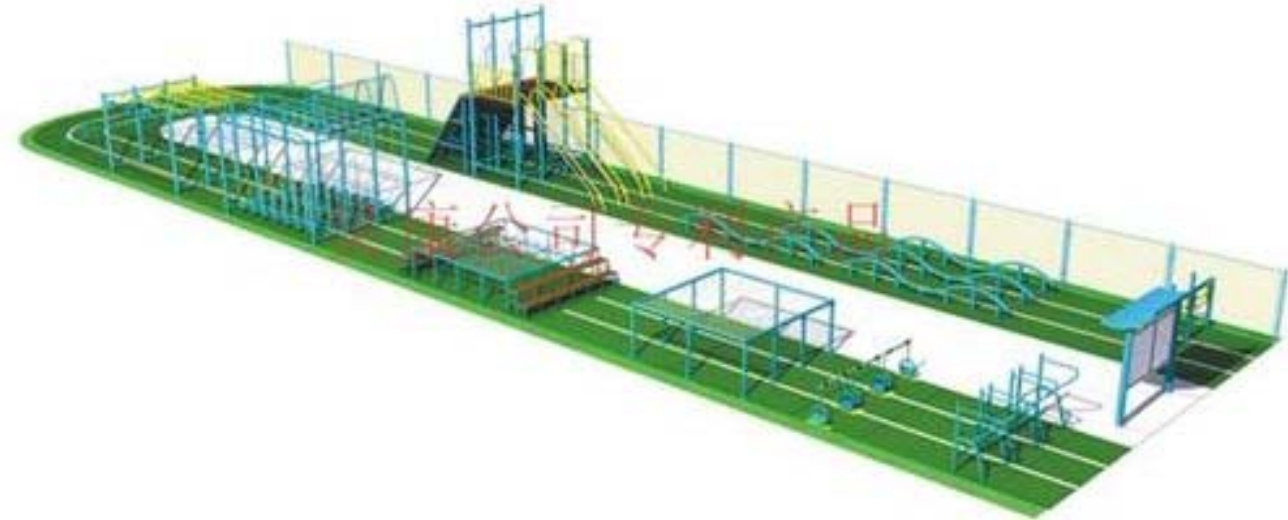

Figure. 1 Closed sports fitness promenade layout

\section{Design of information collection system base on RS485 network}

In order to monitor students' physical exercises participation and the change of students' physical quality in real time, the acquisition of training information in physical exercise must be done to evaluate their physical quality and to guide the students for their exercises.

In order to keep order during the process of enjoying the exercise fitness promenade and develop the comprehensive evaluation of the students' physical quality system, the control access is mounted in the gallery entrance. In an orderly way, the students' in-out of the promenade is scheduled by the central system and conflict is avoided effectively. The photoelectric sensors are 
installed at each entry and exit point of the training items which will record the consumption time that the students pass through it. Data acquisition system will collect and analyze the collected data in student training process. When the trainee leaves the last training items, the screen will show the students' each score of this training and evaluation of his or her physical quality. The system also provides the function of printing all information as you like. The system architecture is shown in Fig.2.

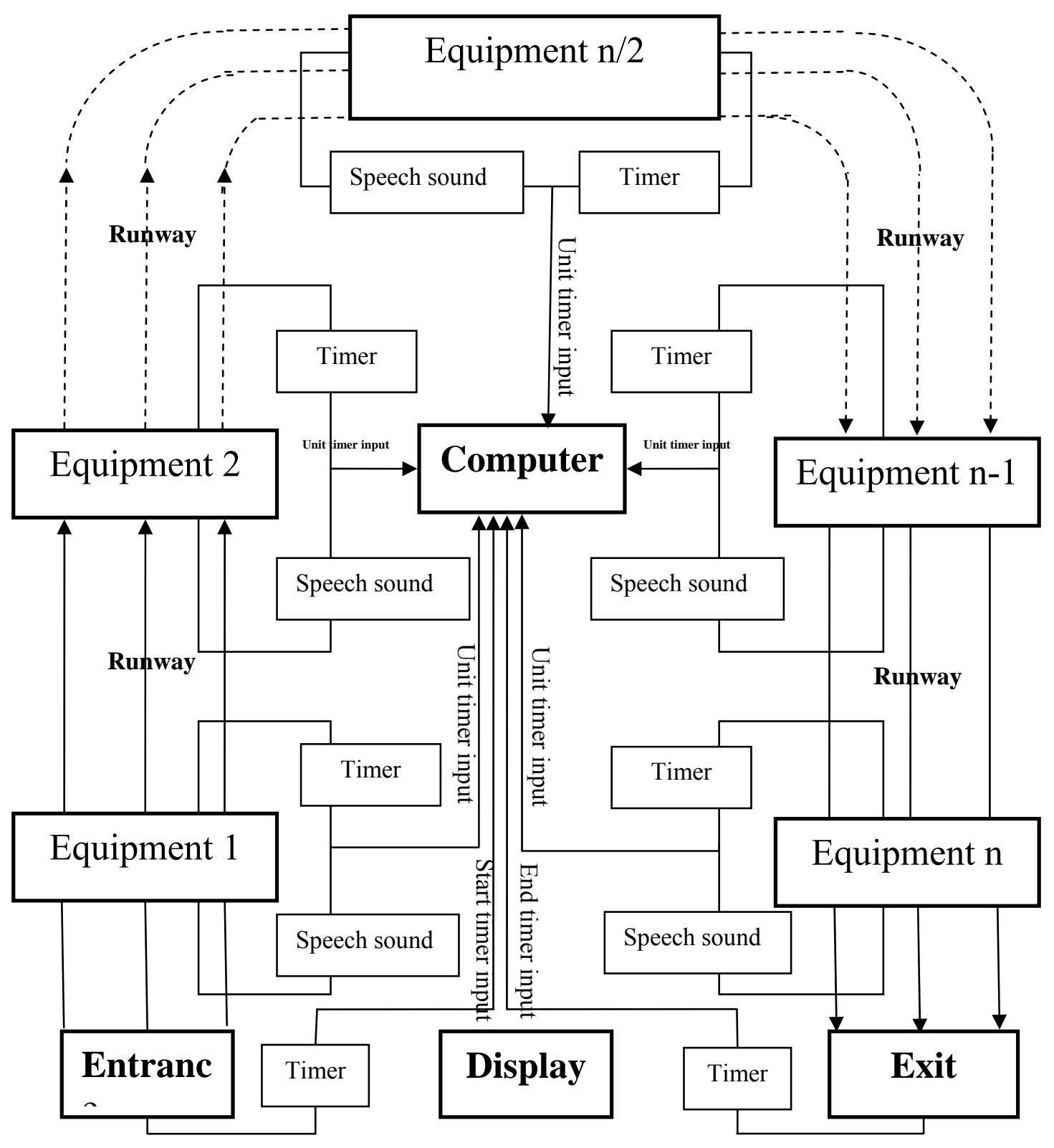

Figure. 2 The system architecture of data acquisition system

In order to use the distributed multi-point data acquisition, the project constructs data acquisition network based on RS485 network. RS485 network uses a pair of twisted connections which can realize multi-station interconnection and constitute a distributed system easily. It has the advantages of simple equipment, low price, long distance communication and is very suitable for small DCS system network construction. Here, we use the distributed data acquisition and centralized control system architecture. The remote I/O is installed in every relevant section training track to acquire sensor signals. The Modbus TCP protocol is adopted by RS485 network to communicate between each embedded controller installed in relevant training track and the main controller. The scheme can break the main tasks down into various sub-tasks to be performed by each embedded controller which increase the ability of real-time response significantly. 
The remote I/O modules count pulses to store signal, avoid missing effective pulses due to long polling period (sometimes the students go through the sensors too quickly). Adopting multi task concurrent processing mechanism, the multiple threads of real-time data acquisition, communication, data storage, the interrupt event monitoring are run independently which improve the processing ability greatly.

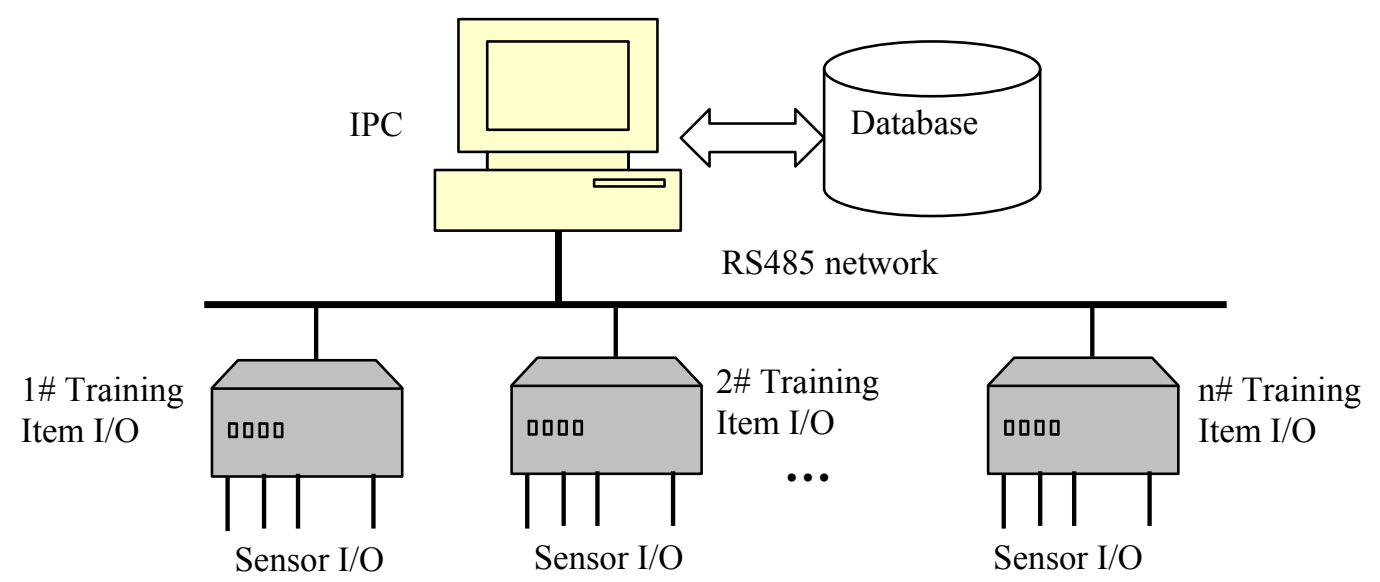

Figure.3 Data Acquisition Network based on RS484

Industrial processing computer is served as the central system which sends the slave I/O commands, interlocks signal, controls the quantities of students flocking into the tracks, and real-time collects the various signals from sensors to monitor the training process information. Finally the training report is generated through the comprehensive evaluation system.

\section{Development of comprehensive evaluation system}

This system could be described as a four element system.

$$
\mathrm{T}(\mathrm{s})=\sum(S, T, I, \Delta t)
$$

Here, $\mathrm{S}$ represents students' information, $\mathrm{T}$ represents time, I represents training item, $\Delta \mathrm{t}$ represents the time consumed by the student to go through specific training item. We build an information database for students to scientifically assess their physical ability, including gender, height and weight. Every time when a student participate in the sports fitness promenade train, the weighting meter will work and update the students' information. The information database of student's training each time is recorded as described in formula (1). Finally, the evaluation information database is built by physical training experts which is described as a five element system, as shown inEq.2.

$$
\mathrm{C}=\sum(G, A, W, I, \Delta t)
$$

In which $\mathrm{G}$ represents gender, $\mathrm{A}$ represents age, $\mathrm{W}$ represent weight, I represent training item, $\Delta \mathrm{t}$ means passing time. It means the expert knowledge database could comprehensively assess students' physical quality according to the students' information such as gender, age, weight, training project and training conditions.

At last, with the help of students' basic information database, training history record database and experts knowledge database, we could come up with the comprehensive assessment of students' physical quality and training instruction by the inference machine. Fig.4 demonstrates its working principle. 


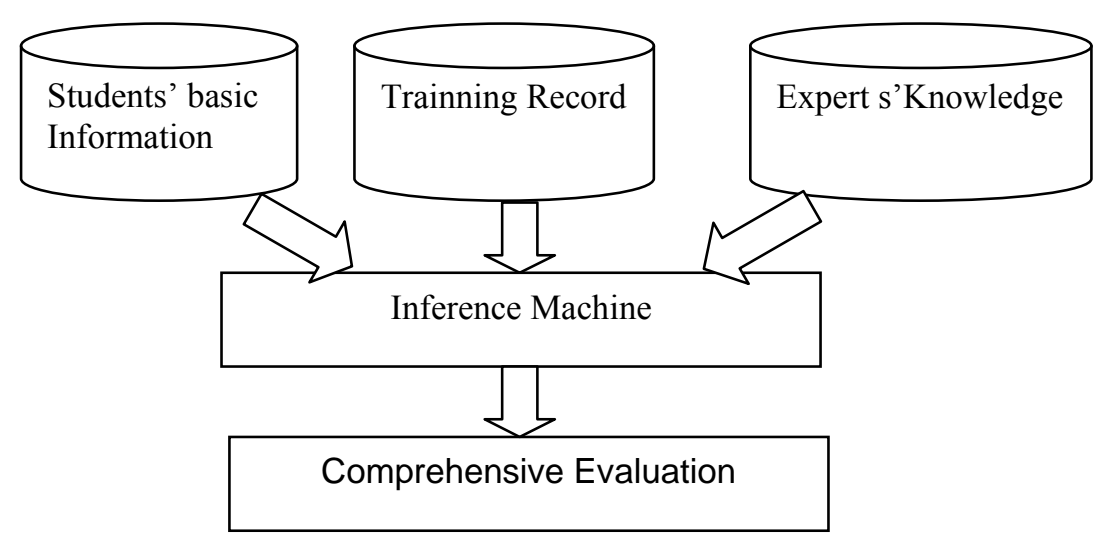

Figure.4 Working mechanism of comprehensive evaluation

Based on the training records, we could analyze the physical condition and fluctuation of the training index for the trainees. Fig.5 gives an example of the data graph based on training records.
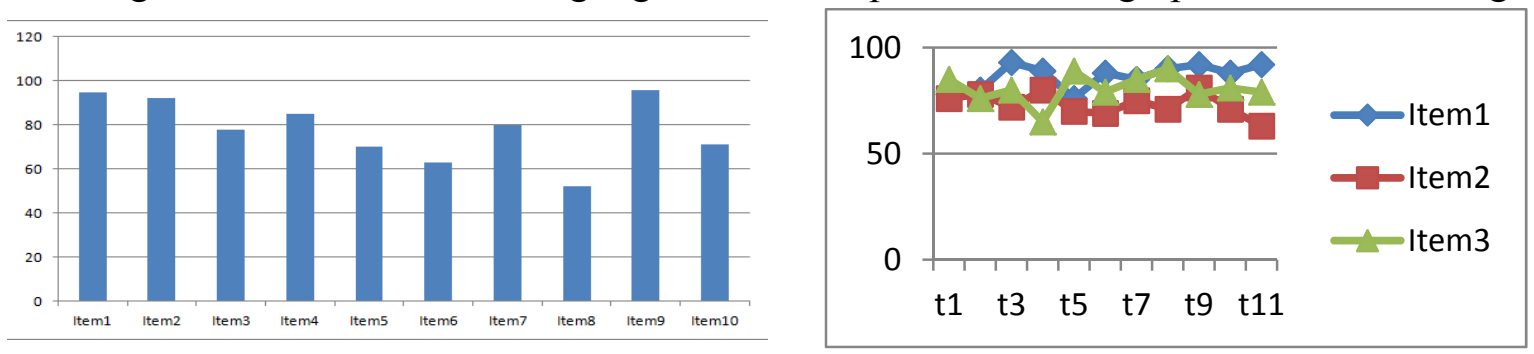

Figure.5 Example of data graph based on training records

\section{Summary}

This paper analyzed the active effect of sunshine sports fitness promenade on students' physical excise. The integrated control and management system was built to acquire the real time information of students' training process and experiment results was given through analyzing the monitoring data. This system has changed the situation of low efficient usage of the traditional sports fitness promenade thoroughly and realized the whole management and control of the physical training data information in school physical exercises. It genuinely realizes the fully implementation of the school physical education, which gains the effectiveness of a continuous development for school physical education. The data acquired in this system could also be use in the physical science study and then could provide theory and practical reference for governmental department and related educational administration.

\section{Acknowledgements}

Supported by

This work was financially supported by the 2012 Chinese Universities Scientific Fund, Humanities and Social Sciences base training project in Donghua University.

\section{References}

[1] Bai FR, Peng YC, Zheng BX. The sunshine sports campus and sports culture exploration in university. [J]. Sports and Science, 2009, 30(2):95-96.

[2] Liu XJ. Explore and analysis "sunshine sports" of the connotation and development.[J]. Journal of Shenyang Institute of Physical Education, 2009, 28(1):25-27. 
[3] Lin SZ, Wu B. Long term mechanism and countermeasures of sunshine sports in China. [J]. Journal of Hebei Institute of Physical Education, 2010,24(1):49-53.

[4] Chen P, Xu GZ, Zhu JY. Effects of enclosed student physical training corridor. [J] on College Students' physical exercise attitude [J]. Journal of Physical Education, 2009, 26(3):55-58.

[5] Xu GZ, Chen P, Zhu JY. Design and development of enclosed student physical training corridor [J]. Shandong Sport Science and Technology, 2007,29(4):55-56.

[6] Xie LX, Xu GZ, Wang ZP. Enclosed student physical training corridor [J]. School physical education, 2007,9:48-49. 